\title{
Evaluation of soft-plaque stenoses in coronary artery stents using conventional and monoenergetic images: first in-vitro experience and comparison of two different dual-energy techniques
}

\author{
Tilman Hickethier ${ }^{1}$, Justus Wenning ${ }^{1}$, Grischa Bratke ${ }^{1}$, David Maintz ${ }^{1}$, Guido Michels ${ }^{2}$, \\ Alexander C. Bunck ${ }^{1}$ \\ ${ }^{1}$ Department of Radiology, ${ }^{2}$ Department III of Internal Medicine, University Hospital Cologne, Cologne, Germany
}

Correspondence to: Tilman Hickethier, MD. University Hospital Cologne, Kerpener Str. 62, 50937 Cologne, Germany. Email: tilman.hickethier@uk-koeln.de.

\begin{abstract}
Background: Non-invasive coronary imaging after stent placement remains challenging. Favorable results for dual-energy computed tomography (DECT) derived monoenergetic (MonoE) images have been reported for this purpose. Nowadays, there are different dual-energy techniques available, each with specific advantages and disadvantages. However, for the evaluation of coronaries after stent implantation there is no systematic comparison between different dual-energy techniques. Therefore, the aim of our study was to compare two widely used DECT systems using an in-stent restenosis (ISR) phantom setup.

Methods: Soft-plaque-like stenoses ( $50 \%$ of lumen) were inserted into ten coronary stents embedded in contrast-filled vessel phantoms. A dual-source CT (DSCT) and a dual-layer CT (DLCT) with comparable acquisition and reconstruction parameters were used. Conventional polyenergetic (PolyE) and MonoE images with 9 different levels $(40-120 \mathrm{keV})$ were calculated. ISR assessability was evaluated by subjective scoring using a 5 -point scale and by the following quantitative parameters: image noise, visible lumen diameter (VLD) and ISR contrast-to-noise ratio (CNR).

Results: A non-significant trend towards larger VLD in DLCT images was observed. Highest noise was found in low-keV MonoE with significantly higher values for DSCT than for DLCT. Conversely, noise was significantly lower for DSCT at higher-keV MonoE. Peak ISR CNR values were found at low-keV MonoE with no significant difference between both systems. However, for PolyE and mid-energy MonoE, CNRs were significantly higher for DSCT. Subjective image quality was significantly better for PolyE and low-keV MonoE than for high-keV MonoE, also without significant difference between both systems.

Conclusions: Conspicuity of ISR benefits from DECT. Peak CNRs were comparable for both DECT systems and low-keV MonoE offered the highest CNR values and best subjective image quality. In contrast, high-keV MonoE cannot be recommended for stent evaluation due to poor CNR values and therefore significantly limited visualization of stenoses.
\end{abstract}

Keywords: Dual energy; computed tomography (CT); stent; cardiac; dual source; dual layer; coronary; in-stent stenosis; artery

Submitted Sep 29, 2019. Accepted for publication Feb 04, 2020.

doi: 10.21037/qims.2020.02.11

View this article at: http://dx.doi.org/10.21037/qims.2020.02.11

\section{Introduction}

Percutaneous coronary interventions are among the most frequently performed medical procedures (1) and in-stent stenosis is the main long-term complication (2). Therefore, reevaluation of the coronary arteries after stent placement is a common task. Despite extensive improvements in computed tomography (CT) technology, the majority of coronary stents is still not sufficiently assessable by CT 
Table 1 Details of the examined stents

\begin{tabular}{|c|c|c|c|c|}
\hline Name & Company & Material & Length (mm) & Strut thickness (mm) \\
\hline Endeavor & Medtronic & $\mathrm{CoCr}$ & 30 & 0.091 \\
\hline Chrono & Sorin Biomedica & $\mathrm{CoCr}$ & 20 & 0.08 \\
\hline Radius & Boston Scientific & Nitinol & 20 & 0.085 \\
\hline Pro Kinetic & Biotronik & $\mathrm{CoCr}$ & 15 & 0.06 \\
\hline Tantal Coronary & Abbott/Guidant & Tantalum & 19 & 0.58 \\
\hline Wiktor & Medtronic & Tantalum & 30 & 0.064 \\
\hline Omega & Boston Scientific & $\mathrm{PICr}$ & 16 & 0.081 \\
\hline
\end{tabular}

imaging (3). As a consequence, coronary CT imaging is currently considered appropriate only for select patients with suspected in-stent stenosis (3-5).

Recent dual energy CT systems offer the option for the reconstruction of additional image types of which monoenergetic (MonoE) images, in particular, have been found to increase image quality and reduce stent associated artifacts $(6,7)$. These stent-associated artifacts are caused by different mechanisms (including beam hardening, scatter effects and Poisson noise), with beam hardening being considered the main factor (8). Therefore, since stentassociated artifacts are the main problem in coronary stent imaging, MonoE images hold promise to be of particular benefit for detecting in-stent stenoses.

In order to generate dual energy CT data different technologies are commercially available, each with certain advantages and disadvantages (9). For the dual-source CT (DSCT) and the dual-layer CT (DLCT), two widespread dual energy CT variants, first studies have confirmed the presumed favorable effects of MonoE reconstructions for coronary stent imaging (10-12). However, these studies focused on the imaging of the stent itself, rather than on the conspicuity of intraluminal alterations. Thus, the influence on the delineation of potential in-stent stenoses and therefore the transferability to clinical practice remains elusive. Additionally, to our knowledge, no systematic comparison between different dual energy CT techniques with respect to coronary stent imaging exists so far.

Hence, the purpose of this in-vitro study was a systematic evaluation of two different dual energy CT scanners with respect to their capability in detecting and delineating instent stenoses.

\section{Methods}

\section{Evaluated stents and experimental setup}

Ten different coronary stents varying in material and stent design (Table 1) with a nominal diameter of $3.0 \mathrm{~mm}$ were studied in an established in-vitro setup (13). Plastic tubes of a material with a CT number comparable to that of a vessel wall $(35 \mathrm{HU})$ and an inner diameter of $3 \mathrm{~mm}$ were used as vessel phantoms. Artificial in-stent stenoses were created using a wax-based material mixed with ethiodized oil (Lipiodol Ultra-Fluid; Guerbet $\mathrm{GmbH}$ ) titrated to a CT number of $45 \mathrm{HU}$ at $120 \mathrm{kVp}$, which is the typical mean density of soft-/lipid-rich-plaques (14-16). After stent deployment the grain-shaped stenoses were positioned inside the stented tube lumen under fluoroscopic guidance. A microwire (V-14 Control Wire; Boston Scientific GmbH) was used to carefully pass the stenosis and to insert a $1.5-\mathrm{mm}$ balloon-catheter (Armada 14; Abbott GmbH). The ballooncatheter was inflated to its nominal pressure to ensure reproducible, wall-adherent artificial $50 \%$ in-stent stenosis (Figure 1). Afterwards, the tubes were filled with Iohexol (300 mg Iohexol/mL; Accupaque 300; GE Healthcare $\mathrm{GmbH}$ ) diluted with saline solution to a CT number of $400 \mathrm{HU}$ (at $120 \mathrm{kVp} / 100 \mathrm{mAs}$ ) and sealed at both ends. For the CT scans, the tubes were separately placed in a fluidfilled plastic container $(36 \times 24 \mathrm{~cm})$ and positioned parallel to the $\mathrm{z}$-axis in the isocenter of the CT scanner. 

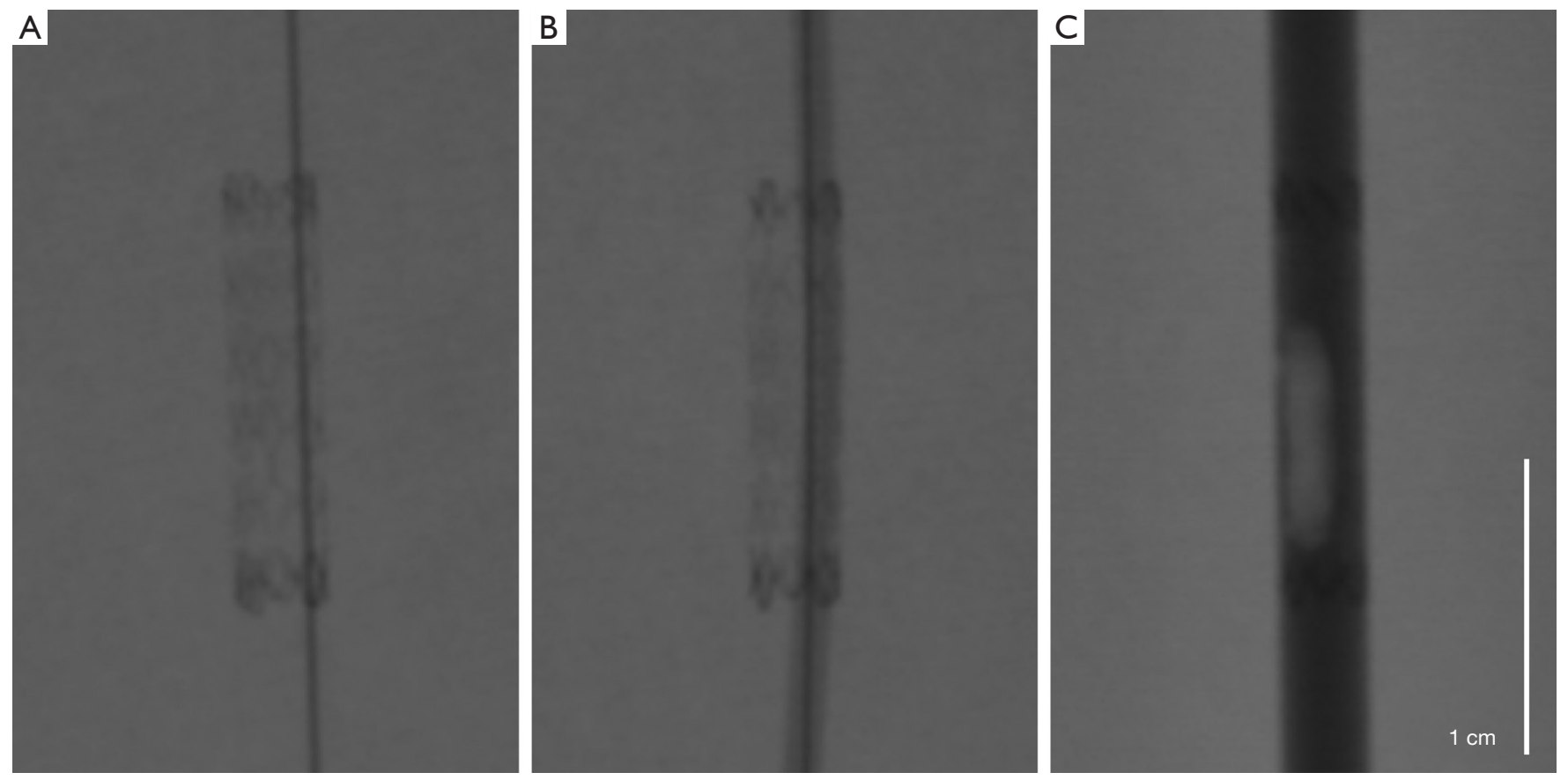

Figure 1 Preparation of stents with artificial in-stent stenoses. (A) Stent and stenoses were positioned and passed with a microwire and 1.5 -mm balloon-catheter; (B) balloon-catheter was inflated to its nominal pressure; (C) angiographical verification of the wall-adherent in-stent stenosis (slightly rotated projection).

\section{CT acquisition and reconstruction parameters}

CT data was acquired on a 64-row DLCT scanner (IQon; Philips GmbH) and a 2×96-row dual-source CT scanner (SOMATOM Force; Siemens Healthcare GmbH). A simulated electrocardiogram (ECG) signal at a heart rate of 60 beats/min was used for prospectively ECGtriggered data acquisition. Tube voltages and currents were adjusted to generate a resulting volume-based CT dose index (CTDIvol) of $9.0 \mathrm{mGy}$ at both systems. Acquisition parameters were chosen as comparable as possible and all modulation and postprocessing processes were deactivated. For image reconstruction of the dual energy raw data kernels (DSCT: Bv49, DLCT: CD) were used which previously have been recommended for stent imaging $(10,11)$ and offered a comparable image noise in our conventional reconstructions (see results). All acquisition and reconstruction parameters are given in Table 2. Conventional polyenergetic (PolyE) images $(120 \mathrm{kVp})$ as well as virtual MonoE images with 9 different energy levels $(40,50,60,70,80,90,100,110,120 \mathrm{keV})$ were calculated. For PolyE images fixed window settings (width 1,500 HU, center $300 \mathrm{HU}$ ) were used as previously recommended (17).
Because contrast conditions vary significantly between the different MonoE levels (18), individually adjusted windows settings were necessary for the MonoE images. For this purpose, a standardized procedure was defined, where first the window center and then the window width was chosen in an optimal way to evaluate the stent lumen (comparable to the procedure in routine clinical practice). The window settings determined by this method were then used by both readers.

\section{CT data analysis}

All quantitative analysis was performed by an experienced reader (6 years of CT reading experience) on a separate offline workstation (IntelliSpace Portal; Philips GmbH). Subjective visibility of the stenosis as well as diameter measurements were additionally performed by a second reader blinded to the results of the first reader after a joint training session. All reconstructions were presented to the readers in a random order and with blinding of the respective acquisition and reconstruction parameters. All measurements were performed at predefined positions.

For evaluation of the visible lumen diameter additional 
Table 2 Used acquisition and reconstruction parameters

\begin{tabular}{lcc}
\hline Variables & DSCT & DLCT \\
\hline Scan type & Axial & Axial \\
Collimation & $2 \times 96 \times 0.6 \mathrm{~mm}$ & $64 \times 0.625 \mathrm{~mm}$ \\
Slice thickness & $0.70 \mathrm{~mm}$ & $0.67 \mathrm{~mm}$ \\
Increment & $0.35 \mathrm{~mm}$ & $0.35 \mathrm{~mm}$ \\
Rotation time & $0.25 \mathrm{~s}$ & $0.27 \mathrm{~s}$ \\
Voltage & Tube A: $90 \mathrm{kV} /$ Tube B: Sn150 kV & $120 \mathrm{kV}$ \\
Current & Tube A: $126 \mathrm{mAs} / \mathrm{Tube} \mathrm{B}: 97 \mathrm{mAs}$ & $100 \mathrm{mAs}$ \\
Dose modulation & None & None \\
CTDlvol & $9.0 \mathrm{mGy}$ & $9.0 \mathrm{mGy}$ \\
Image matrix & $512 \times 512$ & $512 \times 512$ \\
Field of view & $102 \mathrm{~mm}$ & $102 \mathrm{~mm}$ \\
Reconstruction filter & Bv49 (standard VMI) & CD \\
Iterative reconstruction & None & As low as possible (iDose/spectral level 0) \\
\hline
\end{tabular}

DSCT, dual-source computed tomography; DLCT, dual-layer computed tomography; VMI, virtual monoenergetic imaging.

longitudinal multiplanar reformations (MPR) along the stent were reconstructed with a thickness equal to that of the underlying slice stack. The diameter was then measured in the non-stenosed stent segment adjacent to the stenosis with an electronic caliper tool in axial and longitudinal reformatations. After demonstrating good agreement between the measurements obtained by both readers, all measurements for each stent were averaged for further statistical comparison between different reconstructions and CT systems.

The density of the lumen was measured at 3 positions: in the tube outside the stent, in the tube inside the stent distant to the stenosis, in the tube inside the stent at the location of the stenosis. All measurements were performed in 3 consecutive axial images and averaged. The stenosis density difference was calculated as the attenuation (in $\mathrm{HU}$ ) at the location of the stenosis (inside the stent) subtracted by the attenuation inside the non-stenosed stent segment.

Image noise was defined as standard deviation (SD) of CT numbers in a standardized region of interest (ROI) $\left(300 \mathrm{~mm}^{2}\right)$ placed in the fluid of the container adjacent to the stents. The contrast-to-noise ratio (CNR) of the stenosis was calculated as stenosis density difference divided by the image noise (see also Figure $S 1$ for an example image).
Additionally, the subjective delineation of the stenosis and the residual lumen was evaluated on axial and longitudinal MPR images by two readers using a 5-point Likert scale (1: stent associated artifacts impede lumen assessment and stenosis not visible; 2: lumen appears stenosed with unclear longitudinal extent; 3: stenosis and longitudinal extent clear, but residual lumen undistinguishable; 4: stenosis clear and residual lumen slightly distinguishable; 5 : stenosis and residual lumen clear—see also Figure $S 2$ for example images).

\section{Statistical analysis}

Statistical analysis was performed using GraphPad Prism (GraphPad Prism version 8.0.0 for MacOS, GraphPad Software). All data are given as mean \pm SD. Testing for significant differences of continuous data was performed using one-way analysis of variance followed by Tukey multiple comparisons post hoc tests. To compare the subjective image scores Friedman tests followed by Dunn's multiple comparisons post hoc tests were performed. Statistical significance was defined as $\mathrm{P}$ values $<0.05$. The interreader agreement for the visible lumen diameter measurements was calculated using Pearson correlation coefficient and for the subjective image scores by using Cohen's kappa. The values were interpreted according to Landis and Koch (19). 


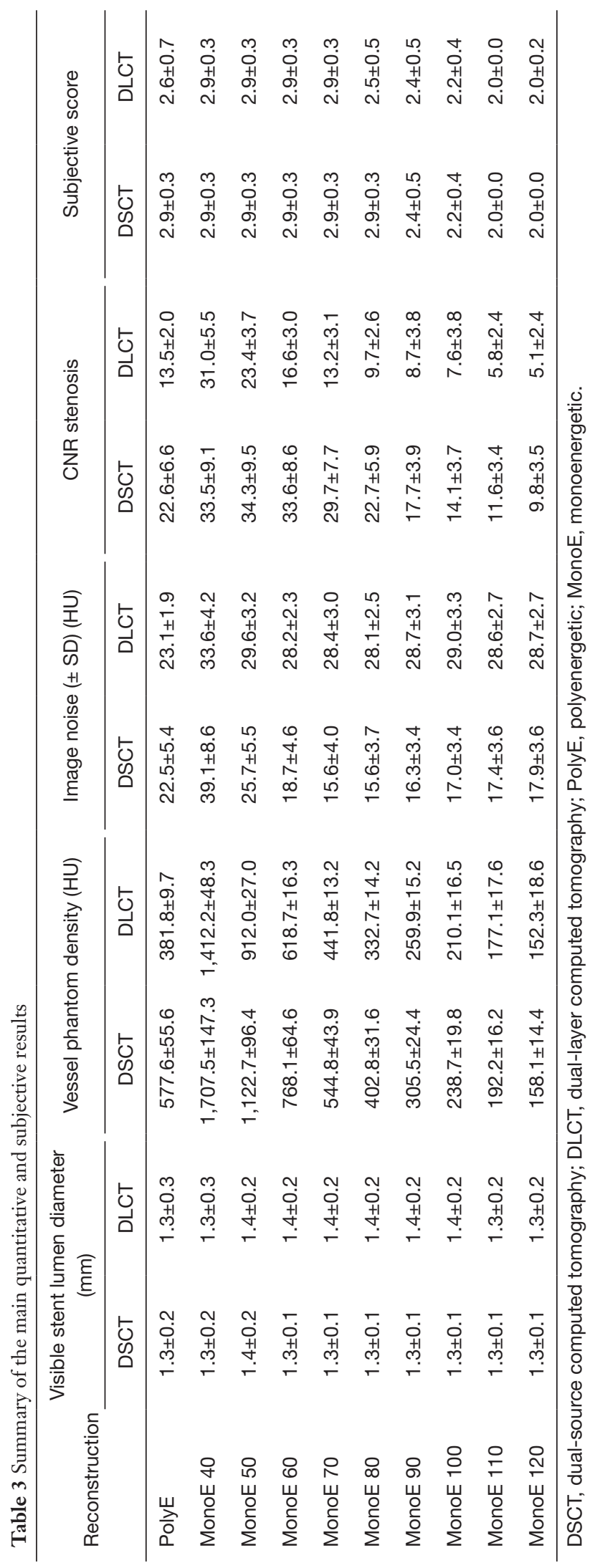

\section{Results}

\section{Visible lumen diameter of the non-stenosed stent lumen}

The interreader agreement for visible lumen diameter measurements in the non-stenosed stent segment was excellent in both, axial (0.85) and longitudinal planes (0.84). There were no significant differences of the mean visible lumen diameters between the different reconstructions of each CT scanner or between the corresponding reconstructions of the two different $\mathrm{CT}$ scanners (all $\mathrm{P} \geq 0.05$ ). However, a trend towards slightly larger visible lumen diameter in DLCT images could be observed throughout all different reconstructions (Table 3, Figure 2).

\section{Density measurements}

The density of the vessel lumen outside of the stent differed significantly between the reconstructions at low energies for both CT systems (40 to $60 \mathrm{keV}, \mathrm{P}<0.05$ ). Conversely, when comparing PolyE and MonoE $70 \mathrm{keV}$ images for the DSCT as well as PolyE and MonoE 70 and $80 \mathrm{keV}$ images for the DLCT and among the higher keV MonoE images (DSCT: 90 to $120 \mathrm{keV}$, DLCT: 80 to $120 \mathrm{keV}$ ) no significant differences were found. Attenuation was highest in MonoE $40 \mathrm{keV}$ images and lowest in MonoE $120 \mathrm{keV}$ images. Also, attenuation was significantly higher in PolyE and MonoE 40 to $80 \mathrm{keV}$ DSCT images than in the corresponding DLCT images (PolyE and MonoE 40 to $70 \mathrm{keV}$ : $\mathrm{P}<0.01$, MonoE $80 \mathrm{keV}: \mathrm{P}=0.03$ ) (Table 3, individual levels of significance not shown separately).

Measurements of the density inside the stented lumen as well as of the stenosis showed similar relations of attenuation values between the different energy levels and both CT systems. Due to stent-related beam hardening the mean attenuation was higher inside the stented lumen (mean attenuation for PolyE DSCT 586.2 $\pm 96.8 \mathrm{HU}$ and DLCT $531.5 \pm 168$.9 HU), while it was reduced inside the stenoses (mean attenuation for PolyE DSCT $111.3 \pm 74.9 \mathrm{HU}$ and DLCT 220.4 $\pm 150.6 \mathrm{HU}$ ) (see Table $S 1$ for detailed results).

\section{Image noise}

For DSCT, image noise was significantly higher for MonoE $40 \mathrm{keV}$ images $(\mathrm{P}<0.01)$ and significantly lower for MonoE 70,80 and $90 \mathrm{keV}$ images $(\mathrm{P}=0.01 / 0.01 / 0.04)$, when compared to PolyE images. Among the DSCT MonoE reconstructions, 40 and $50 \mathrm{keV}$ images showed significantly higher image noise than all other DSCT MonoE images 


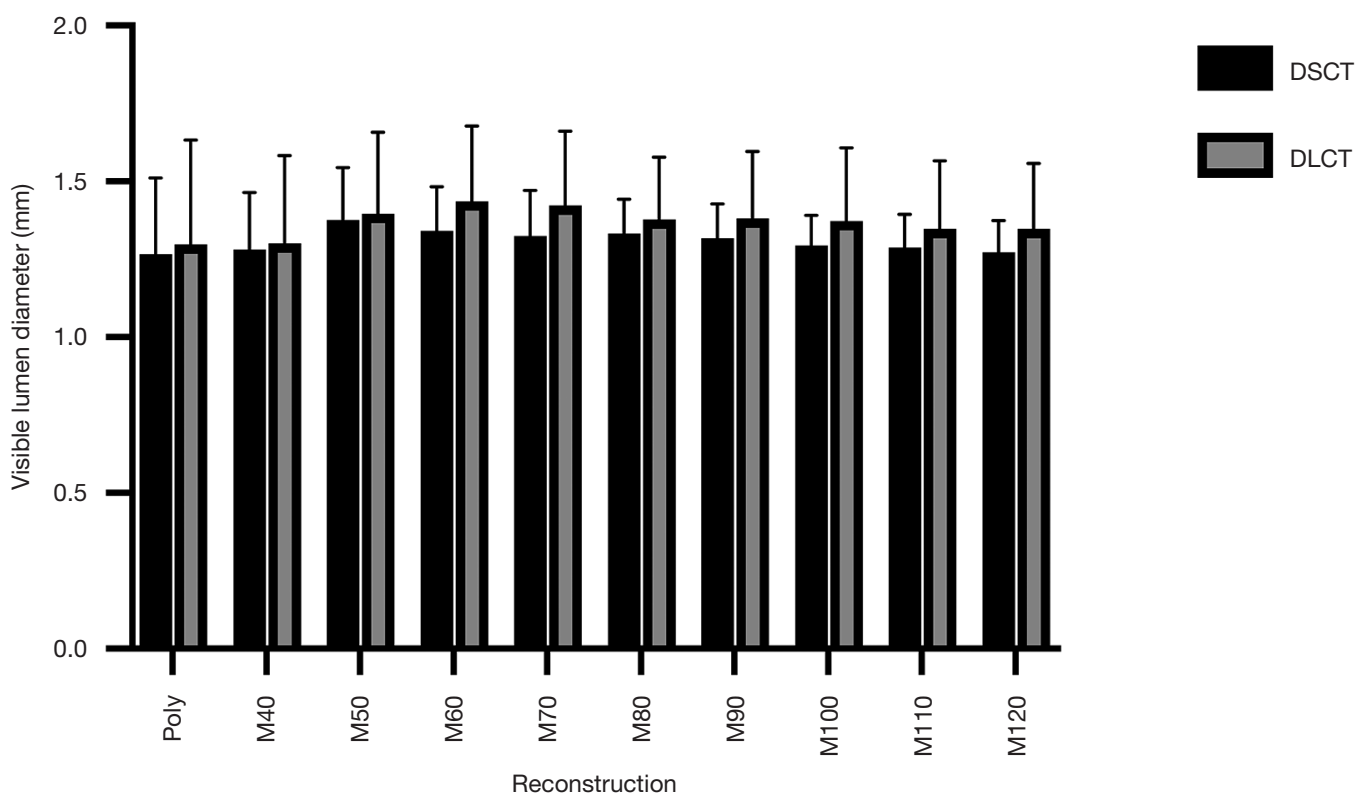

Figure 2 Graphical depiction of the visible lumen diameter (higher is superior; error bars indicate the standard deviation) for the different reconstructions of the two different CT scanners. There were no significant differences found between the different reconstructions or between corresponding reconstructions of the two different CT scanners. Poly, conventional polyenergetic images; M, virtual monoenergetic images; the number indicates the keV level; DSCT, dual-source computed tomography; DLCT, dual-layer computed tomography.

(all $\mathrm{P} \leq 0.01$ ). For DLCT, variations of image noise among the different MonoE levels were smaller. In MonoE 40 and $50 \mathrm{keV}$ images image noise was significantly higher than in DLCT PolyE images $(\mathrm{P}<0.01, \mathrm{P}=0.03$ respectively). Among all other DLCT reconstructions no significant differences were found.

There was no significant difference in image noise between DSCT and DLCT PolyE images $(\mathrm{P}>0.05)$. For MonoE $40 \mathrm{keV}$ images, noise was significantly lower in DLCT than in DSCT data $(\mathrm{P}=0.04)$, whereas for MonoE $60 \mathrm{keV}$ and higher energy levels noise was significantly lower in DSCT than in DLCT data (all $\mathrm{P}<0.01)$ (Table 3).

\section{Contrast to noise ratios between the stenosed and non- stenosed stent lumen}

Based on these image parameter results (see Table $S 1$ for detailed results) the CNRs between the stenosed and nonstenosed stent lumen at the different energy levels were calculated for both CT systems (see Figure 3). For both, DSCT and DLCT, low $\mathrm{keV}$ MonoE reconstructions (DSCT 40-60 keV, DLCT 40 and $50 \mathrm{keV}$ ) offered significantly higher CNR values than PolyE images (all $\mathrm{P}<0.01)$. High $\mathrm{keV}$ MonoE reconstructions on the other hand (DSCT 100-120 keV, DLCT $120 \mathrm{keV}$ ) provided significantly lower CNR values than the PolyE images (all $\mathrm{P} \leq 0.03)$. CNR was significantly higher in PolyE and midenergy MonoE (50-90 keV) DSCT reconstructions than in the corresponding DLCT images (all $\mathrm{P} \leq 0.01$ ).

The highest CNRs were found at MonoE $50 \mathrm{keV}$ for DSCT and MonoE $40 \mathrm{keV}$ for DLCT, with no significant difference between both CT systems $(\mathrm{P}>0.05)$ (Table 3).

With respect to the density values of the stenotic region, an increase in density in the low $\mathrm{keV}$ MonoE reconstructions compared to the PolyE and the high $\mathrm{keV}$ MonoE images was observed, but this was markedly less pronounced than the increase in density in the rest of the stent lumen, resulting in enhanced CNR values as described above (see Table $S 1$ and Figure $S 3$ for detailed results).

\section{Qualitative assessment of stenosis visibility}

Accordingly, subjective visibility of the stenoses in PolyE and low MonoE reconstructions was superior to high $\mathrm{keV}$ MonoE reconstructions. For DSCT, best subjective visibility of the stenoses was found for PolyE and MonoE 40 to $80 \mathrm{keV}$ with significant higher scores than for MonoE 110 and $120 \mathrm{keV}$ images (all $\mathrm{P}<0.05$ ). For DLCT, best 


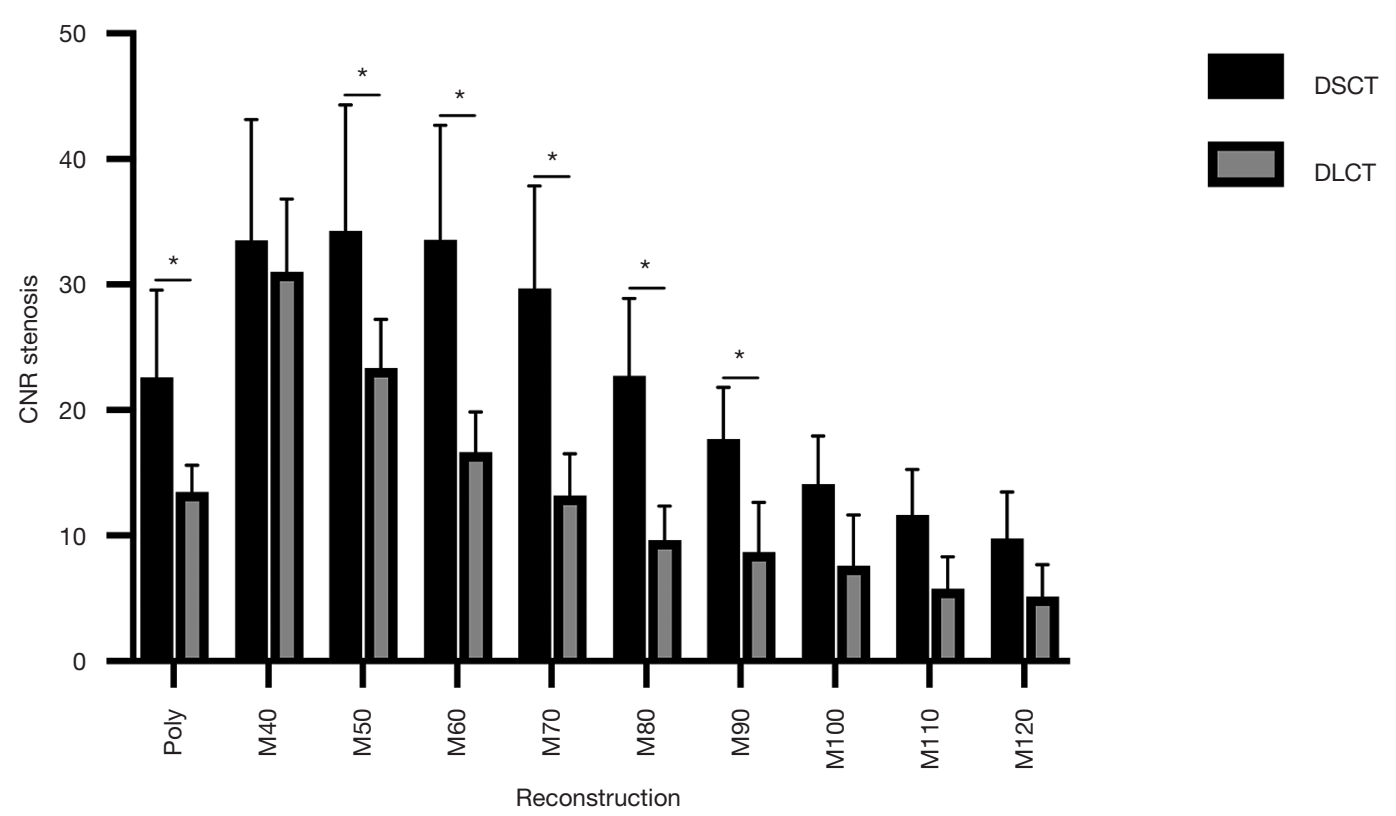

Figure 3 Graphical depiction of the contrast-to-noise ratio of the stenosis (higher is superior; error bars indicate the standard deviation) for the different reconstructions of the two different CT scanners. Asterisks indicate significant differences between the corresponding reconstructions of the two different CT systems. Between the different reconstructions of the respective CT systems the following significant differences were found (we dispensed with the graphical depiction of the individual significances between the reconstructions in the figure, as this is not comprehensibly feasible due to their large number): DSCT: Poly vs. M40, M50, M60, M100, M110, M120/ M40 vs. M80-120/M50 vs. M80-120/M60 vs. M80-120/M70 vs. M80-120/M80 vs. M100-120; DLCT: Poly vs. M40, M50, M120/M40 vs. M60-120/M50 vs. M70-120/M60 vs. M90-120/M70 vs. M120. CNR, contrast-to-noise ratio; Poly, conventional polyenergetic images; M, virtual monoenergetic images; the number indicates the keV level; DSCT, dual-source computed tomography; DLCT, dual-layer computed tomography.

subjective visibility of the stenoses was found for MonoE 40 to $70 \mathrm{keV}$ with significant higher scores than for MonoE 110 and $120 \mathrm{keV}$ images (all $\mathrm{P}<0.05$ ). There were no significant differences among the corresponding DSCT and DLCT images (see Figure 4). The interreader agreement for the subjective evaluation of stenosis visibility was excellent (0.97).

\section{Discussion}

This study is the first systematic comparison of two different dual energy CT techniques for non-invasive coronary stent imaging. By using artificial in-stent stenoses we aimed at creating an in-vitro setup, which reflects the clinical challenge of detecting in-stent stenosis.

For a fair comparison between the DLCT and the DSCT technology, acquisition and reconstruction parameters were set to be as similar as possible (see Table 2). In particular, acquisition parameters were adjusted in such a way, that images were obtained at equal radiation doses on both systems. However, due to technical constraints, not all parameters could be set to be identical with potential influences on image quality (e.g., different tube potentials provoke different attenuations). Additionally, both systems use different image reconstruction algorithms, which have only partly been disclosed by the vendors. To address this issue, iterative reconstructions and other measures of image enhancement [e.g., noise-optimized virtual monoenergetic imaging (VMI+) (20)] were disabled where possible. We used coronary CT specific kernels, which have been applied in previous CT stent imaging studies $(10,11)$ and resulted in comparable noise levels in conventional reconstructions in our study. Nevertheless, differences with respect to the underlying image reconstruction algorithms remain, especially when considering the different dual-energy postprocessing techniques [image vs. raw data domain (9) for DSCT and DLCT, respectively]. Therefore, comparability between such different systems is always limited and the 


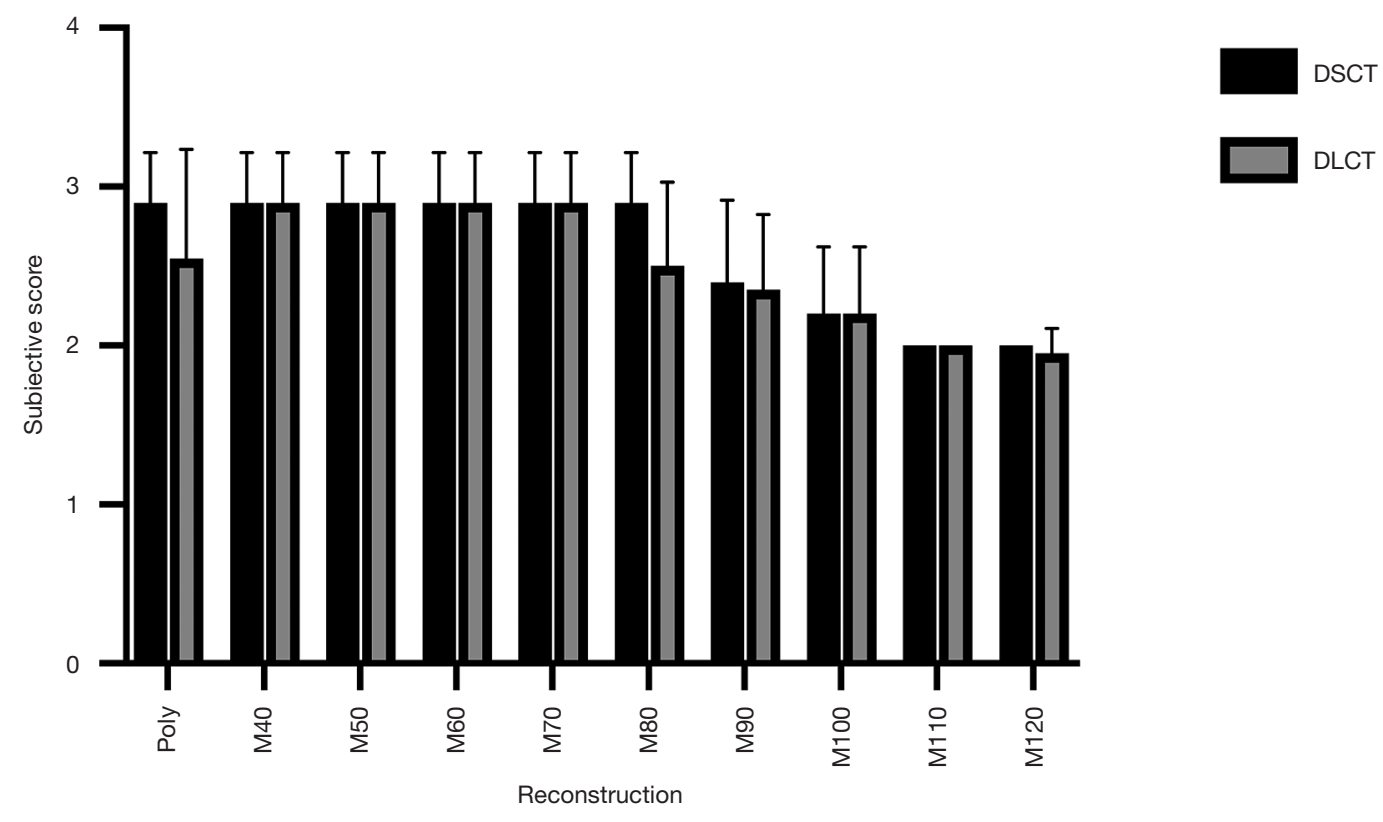

Figure 4 Graphical depiction of the subjective visibility of the stenoses (higher is superior; error bars indicate the standard deviation) for the different reconstructions of the two CT scanners. Between the corresponding reconstructions of the two different CT systems the following significant differences were found (we dispensed with the graphical depiction of the individual significances between the reconstructions in the figure, as this is not comprehensibly feasible due to their large number): DSCT: Poly vs. M110, M120/M40 vs. M110, M120/M50 vs. M110, M120/M60 vs. M110, M120/M70 vs. M110, M120/M80 vs. M110, M120; DLCT: M40 vs. M110, M120/M50 vs. M110, M120/ M60 vs. M110, M120/M70 vs. M110, M120. There were no significant differences found between the corresponding reconstructions of the two different CT scanners. Poly, conventional polyenergetic images; $M$, virtual monoenergetic images; the number indicates the keV level; DSCT, dual-source computed tomography; DLCT, dual-layer computed tomography.

obtained image selection only represents a part of each system's capabilities. Further improvements in image quality can be expected when applying the above-mentioned device/manufacturer-specific optimized reconstruction algorithms, but these were not the subject of this study.

In line with previous observations $(10,11)$, we observed a trend towards larger visual stent lumen diameters in midenergetic MonoE images compared to the PolyE and lowenergetic MonoE images. However, in our current study this trend did not reach significance nor was continued in high-energetic MonoE images. While these findings may appear somewhat contradictory to the abovementioned previously published results, these interstudy differences are most likely related to the slightly different acquisition and reconstructions parameters as well as the different window settings, which were optimized to give a high contrast between the stenosed and non-stenosed in-stent segments in our current study. Additionally, visual lumen diameters were marginally larger in DLCT images, also without reaching significance.
Changes in attenuation and image noise were in line with previous results of both systems $(21,22)$ showing higher image noise and attenuation at low $\mathrm{keV}$ MonoE levels decreasing with increasing $\mathrm{keV}$ levels. While image noise was higher for the DSCT system at $40 \mathrm{keV}$ MonoE, image noise was lower for 60 and higher $\mathrm{keV}$ MonoE reconstructions when compared to the DLCT system.

Beyond a high spatial resolution, the detectability and delineation of in-stent stenosis depends on the CNR between the stenosed and non-stenosed lumen. As a composite image parameter, CNR reflects the attenuation difference between two structures relative to the image noise. In our study, the highest CNR values were found for low $\mathrm{keV}$ MonoE images as the increase in attenuation difference exceeded the increase in image noise. At these low $\mathrm{keV}$ reconstructions, both systems reached comparable CNR values. For mid- and high-MonoE images CNR values were higher for the DSCT than for the DLCT system, primarily due to lower image noise at these energy levels. However, even though high $\mathrm{keV}$ images offer 


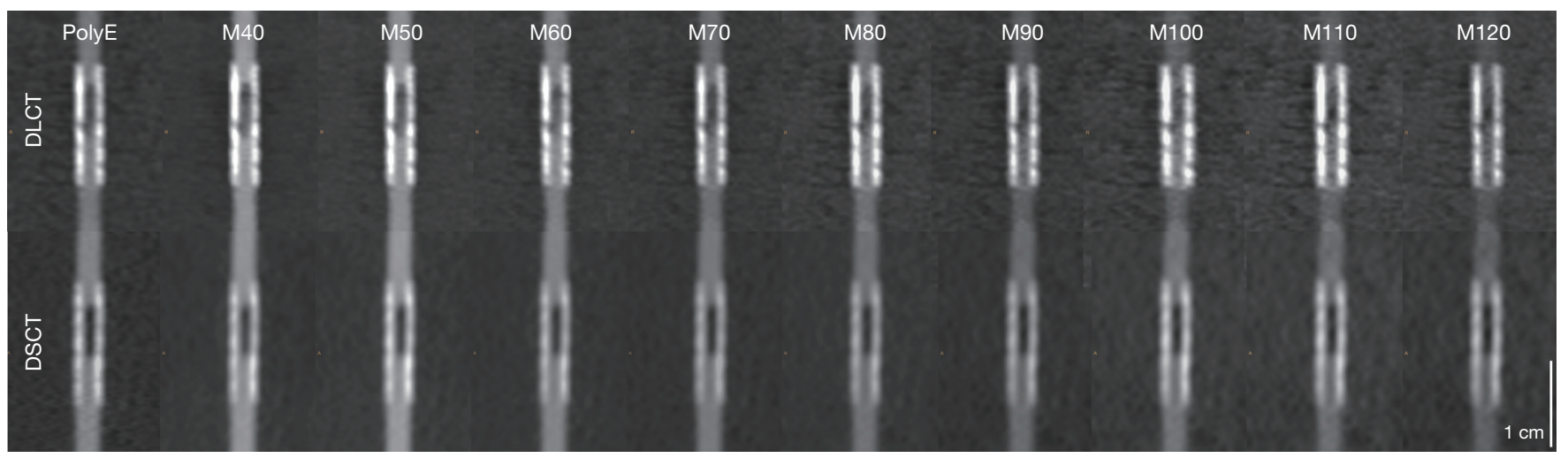

Figure 5 Identical cobalt-chromium stent ("Pro Kinetic"; diameter $3.0 \mathrm{~mm}$, length $15 \mathrm{~mm}$, strut thickness $0.06 \mathrm{~mm}$ ) scanned with both CT systems and reconstructed with different algorithms and settings. Best image quality was observed for low keV monoenergetic images for both scanners, however for DSCT with a broader range of keV levels with favorable image quality. DSCT, dual-source computed tomography; DLCT, dual-layer computed tomography; PolyE, polyenergetic; MonoE, monoenergetic.

decreased image noise and reduce the stent associated artifacts mentioned in the introduction (11), they are also associated with a significant drop in iodine attenuation due to the higher distance to the iodine k-edge at $33 \mathrm{keV}$ (23). This drop leads to a strong decline in CNR between the stenosed and non-stenosed segment. At $120 \mathrm{keV}$ CNR is more than three times lower in DSCT images and even more than six times lower in DLCT when compared to the highest CNR value of each system. These results are in contrast to previous studies $(11,12)$, which have advocated the use of reconstructions at higher energy levels for the benefit of reduced stent associated artifacts and improved lumen visibility but at the same time disregarded the need for a high CNR between the non-stenosed and the stenosed stent section.

The subjective assessment of the stenosis visibility gave concordant results to the CNR values. Thus, highest ratings were achieved for low- and mid-energetic MonoE images, whereas high $\mathrm{keV}$ images were rated significantly lower. There was no significant difference in subjective image quality between both systems.

When planning the study, we expected a better delineation of the residual lumen at the stenosed segment. Accordingly, the Likert scale was defined. However, during the actual evaluation process, scores 4 and 5 , consistent with a clear delineation of the extent of the stenosis and the residual lumen [5] or a vague delineation of both [4], were not assigned once. Additionally, score 1, consistent with an image quality impeding lumen assessment, was also just assigned once. As a result, the differentiation achieved by the used scale was somewhat limited, but the superiority of certain reconstructions was still clearly apparent. Figure 5 shows an example of the same stent with all evaluated reconstructions derived from both CT scanners. The determined visual lumen diameters showed no significant difference. In neither reconstruction of both systems the residual lumen, which was angiographically visible in all stents (see also Figure 1 for comparison), was delineated adequately. This is most likely due to the general trend of an oversized depiction of coronary stenoses in CT imaging (24) adding to the known stent associated artifacts. As indicated by first preliminary reports, novel photon counting detectors hold promise to overcome these limitations and allow for grading of coronary in-stent stenoses for the first time (25).

With regard to image quality, MonoE images at low keV levels provided better subjective ratings and significantly higher CNRs between the stenosed and non-stenosed stent lumen than PolyE and especially high keV MonoE images. In the described setup, both systems' optimal image reconstructions (DSCT: MonoE $50 \mathrm{keV}$, DLCT: MonoE $40 \mathrm{keV}$ ) achieved comparable objective and subjective ratings. However, results for non-ideal reconstructions (PolyE and high keV) tended to be poorer for DLCT images. Also, the range of reconstructions with favorable image quality (e.g., subjective score $\geq 2.5$ and $C N R \geq 20$ ) was smaller for the DLCT system (MonoE 40 and $50 \mathrm{keV}$ images) than for the DSCT system (PolyE and MonoE 40-80 keV images).

The stent manufacturing material is known to have a 


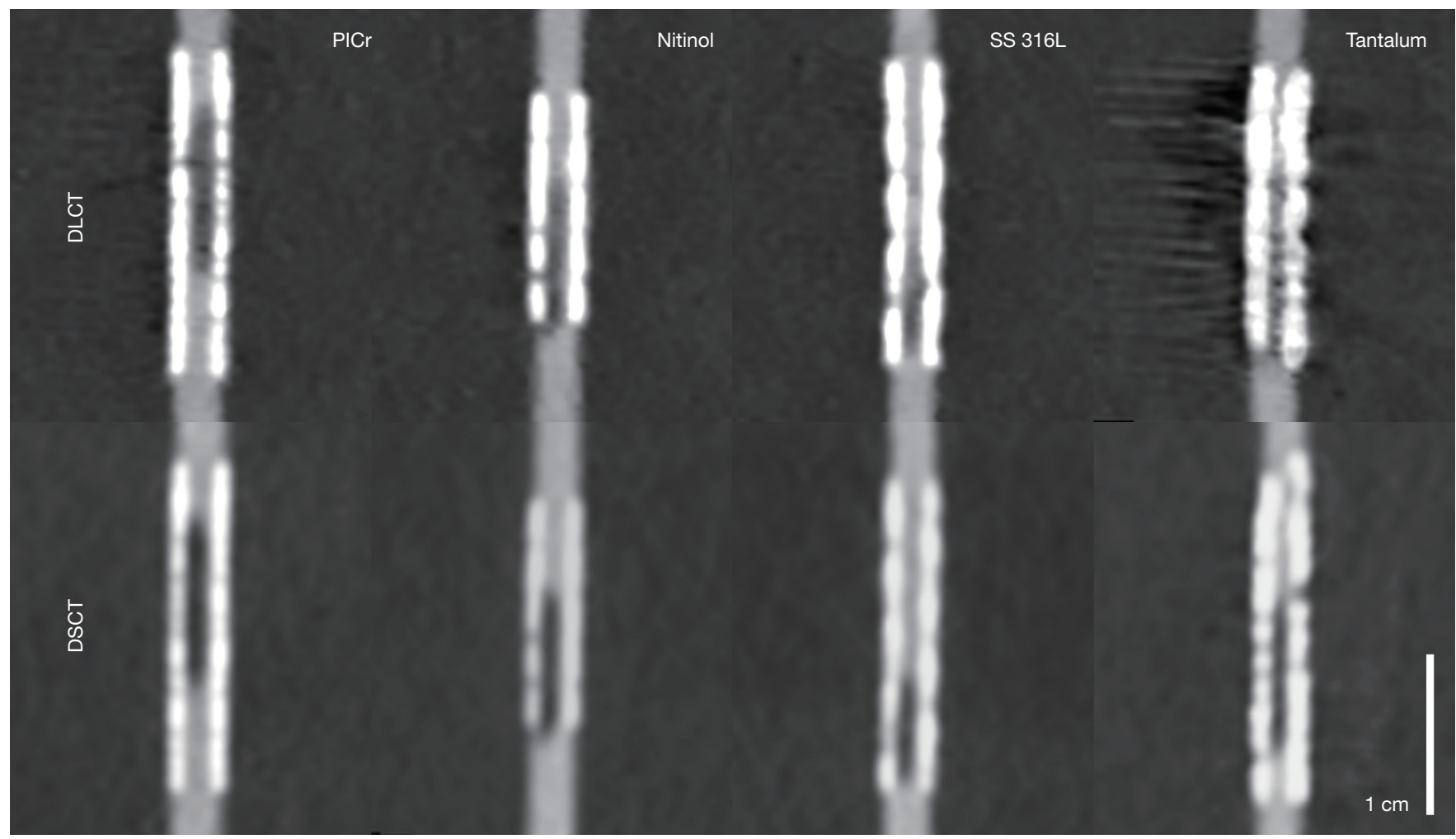

Figure 6 Four stents consisting of different manufacturing materials ("Promus Element Plus", "Radius", "Coroflex Please”, "Tantal Coronary") scanned with both CT systems and reconstructed with MonoE $40 \mathrm{keV}$ illustrating the influence of the individual material on stent related artifacts. Strongest artifacts were found for stents manufactured of tantalum. DSCT, dual-source computed tomography; DLCT, dual-layer computed tomography; PolyE, polyenergetic; MonoE, monoenergetic.

big influence on the stent-associated artifacts and visibility of the stent lumen $(13,26)$. Therefore, we included stents manufactured of all five commonly used materials in our study. Although the limited number of stents of each manufacturing materials is obviously too small for a systematic statistical comparison, the observations of each individual stent type were in good agreement with the above-mentioned previous findings of different stent materials. Accordingly, nitinol and cobalt-chromium caused the least stent-associated artifacts followed by stainless steel and platinum-chromium. As previously described (13), tantalum caused the strongest artifacts. No reconstruction method or dual energy CT technique seemed to be especially advantageous or disadvantageous for any of the manufacturing materials. Figure 6 shows examples for each of the evaluated manufacturing materials for both CTs.

In addition to the already mentioned limitations, there are further aspects to consider in our in-vitro setup. First, cardiac motion was not simulated and therefore possible motion-related artifacts as well as the individual acquisition speed of both systems were not taken into account. Secondly, patient-specific influences (like different heart rates, body constitutions, etc.) and other potentially interacting artifacts were also not included. Still, reconstructions which provided superior image quality in our in-vitro setup are also likely to be favorable in the clinical setting. As extensively discussed above, the comparability of both systems is limited due to the technical constraints in aligning acquisition and reconstruction parameters.

\section{Conclusions}

Non-invasive coronary stent imaging for the detection of in-stent stenoses remains challenging. In our study, we were able to show that coronary stent CT imaging and stenosis detection benefits from dual energy techniques. Both tested CT systems exhibited significantly increased CNR values 
for in-stent stenoses at lower MonoE values allowing for a reliable detection of stenoses. While optimal CNR levels did not differ between both systems, the DSCT system excelled in this respect over a large range of energy levels. On our 5-point Likert scale, however, the increased CNR did not translate into significantly improved subjective scores when comparing lower MonoE to PolyE images. Even though conspicuity of in-stent stenosis benefits from lower $\mathrm{keV}$ reconstructions and may thus increase the diagnostic certainty in the detection of stenosis, none of the reconstructions of either CT system allowed for delineation of the residual lumen. In a clinical scenario any suspicion of in-stent stenoses on a CT image would thus necessitate further workup by invasive angiography to test for hemodynamic significance.

\section{Acknowledgments}

Funding: None.

\section{Footnote}

Conflicts of Interest: The authors have no conflicts of interest to declare.

Open Access Statement: This is an Open Access article distributed in accordance with the Creative Commons Attribution-NonCommercial-NoDerivs 4.0 International License (CC BY-NC-ND 4.0), which permits the noncommercial replication and distribution of the article with the strict proviso that no changes or edits are made and the original work is properly cited (including links to both the formal publication through the relevant DOI and the license). See: https://creativecommons.org/licenses/by-nc-nd/4.0/.

\section{References}

1. Stefanini GG, Holmes DR. Drug-Eluting CoronaryArtery Stents. N Engl J Med 2013;368:254-65.

2. Lagerqvist B, James SK, Stenestrand U, Lindbäck J, Nilsson T, Wallentin L. Long-Term Outcomes with Drug-Eluting Stents versus Bare-Metal Stents in Sweden. N Engl J Med 2007;356:1009-19.

3. Schroeder S, Achenbach S, Bengel F, Burgstahler C, Cademartiri F, de Feyter P, George R, Kaufmann P, Kopp AF, Knuuti J, Ropers D, Schuijf J, Tops LF, Bax JJ; Working Group Nuclear Cardiology and Cardiac CT; European Society of Cardiology; European Council of
Nuclear Cardiology. Cardiac computed tomography: indications, applications, limitations, and training requirements: report of a Writing Group deployed by the Working Group Nuclear Cardiology and Cardiac CT of the European Society of Cardiology and the European Council of Nuclear Cardiology. Eur Heart J 2008;29:531-56.

4. Authors/Task Force members, Windecker S, Kolh P, Alfonso F, Collet JP, Cremer J, Falk V, Filippatos G, Hamm C, Head SJ, Jüni P, Kappetein AP, Kastrati A, Knuuti J, Landmesser U, Laufer G, Neumann FJ, Richter DJ, Schauerte P, Sousa Uva M, Stefanini GG, Taggart DP, Torracca L, Valgimigli M, Wijns W, Witkowski A. 2014 ESC/EACTS Guidelines on myocardial revascularization: The Task Force on Myocardial Revascularization of the European Society of Cardiology (ESC) and the European Association for Cardio-Thoracic Surgery (EACTS) Developed with the special contribution of the European Association of Percutaneous Cardiovascular Interventions (EAPCI). Eur Heart J 2014;35:2541-619.

5. Intracoronary stent restenosis - UpToDate. Available online: https://www.uptodate.com/contents/ intracoronary-stent-restenosis? search=Intracoronary $\% 20$ stent\%20restenosis\&source=search result\&selected Title=1 21\&usage_type=default $\&$ display_ rank=1. Accessed January 19, 2019.

6. Lewis M, Reid K, Toms AP. Reducing the effects of metal artefact using high $\mathrm{keV}$ monoenergetic reconstruction of dual energy CT (DECT) in hip replacements. Skeletal Radiol 2013;42:275-82.

7. Laukamp KR, Lennartz S, Neuhaus VF, Große Hokamp N, Rau R, Le Blanc M, Abdullayev N, Mpotsaris A, Maintz $\mathrm{D}$, Borggrefe J. CT metal artifacts in patients with total hip replacements: for artifact reduction monoenergetic reconstructions and post-processing algorithms are both efficient but not similar. Eur Radiol 2018;28:4524-33.

8. Barrett JF, Keat N. Artifacts in CT: Recognition and Avoidance. RadioGraphics 2004;24:1679-91.

9. McCollough CH, Leng S, Yu L, Fletcher JG. Dual- and Multi-Energy CT: Principles, Technical Approaches, and Clinical Applications. Radiology 2015;276:637-53.

10. Mangold S, Cannaó PM, Schoepf UJ, Wichmann JL, Canstein C, Fuller SR, Muscogiuri G, Varga-Szemes A, Nikolaou K, De Cecco CN. Impact of an advanced image-based monoenergetic reconstruction algorithm on coronary stent visualization using third generation dualsource dual-energy CT: a phantom study. Eur Radiol 2016;26:1871-8. 
11. Hickethier T, Baeßler B, Kroeger JR, Doerner J, Pahn G, Maintz D, Michels G, Bunck AC. Monoenergetic reconstructions for imaging of coronary artery stents using spectral detector CT: In-vitro experience and comparison to conventional images. J Cardiovasc Comput Tomogr 2017;11:33-9.

12. Stehli J, Fuchs TA, Singer A, Bull S, Clerc OF, Possner M, Gaemperli O, Buechel RR, Kaufmann PA. First experience with single-source, dual-energy CCTA for monochromatic stent imaging. Eur Heart J Cardiovasc Imaging 2015;16:507-12.

13. Maintz D, Seifarth H, Raupach R, Flohr T, Rink M, Sommer T, Ozgün M, Heindel W, Fischbach R. 64-slice multidetector coronary CT angiography: in vitro evaluation of 68 different stents. Eur Radiol 2006;16:818-26.

14. Schroeder S, Kuettner A, Leitritz M, Janzen J, Kopp AF, Herdeg C, Heuschmid M, Burgstahler C, Baumbach A, Wehrmann M, Claussen CD. Reliability of differentiating human coronary plaque morphology using contrastenhanced multislice spiral computed tomography: a comparison with histology. J Comput Assist Tomogr 2004;28:449-54.

15. Galonska M, Ducke F, Kertesz-Zborilova T, Meyer R, Guski H, Knollmann FD. Characterization of Atherosclerotic Plaques in Human Coronary Arteries With 16-Slice Multidetector Row Computed Tomography by Analysis of Attenuation Profiles. Acad Radiol 2008;15:222-30.

16. Becker CR, Nikolaou K, Muders M, Babaryka G, Crispin A, Schoepf UJ, Loehrs U, Reiser MF. Ex vivo coronary atherosclerotic plaque characterization with multidetector-row CT. Eur Radiol 2003;13:2094-8.

17. Seifarth H, Ozgün M, Raupach R, Flohr T, Heindel W, Fischbach R, Maintz D. 64- Versus 16-slice CT angiography for coronary artery stent assessment: in vitro experience. Invest Radiol 2006;41:22-7.

18. Doerner J, Luetkens JA, Iuga AI, Byrtus J, Haneder S, Maintz D, Hickethier T. Poly-energetic and virtual monoenergetic images from a novel dual-layer spectral detector CT: optimization of window settings is crucial to improve subjective image quality in abdominal CT angiographies. Abdom Radiol (NY) 2018;43:742-50.

19. Landis JR, Koch GG. The measurement of observer agreement for categorical data. Biometrics 1977;33:159-74.
20. De Cecco CN, Caruso D, Schoepf UJ, De Santis D, Muscogiuri G, Albrecht MH, Meinel FG, Wichmann JL, Burchett PF, Varga-Szemes A, Sheafor DH, Hardie AD. A noise-optimized virtual monoenergetic reconstruction algorithm improves the diagnostic accuracy of late hepatic arterial phase dual-energy CT for the detection of hypervascular liver lesions. Eur Radiol 2018;28:3393-404.

21. Yu L, Christner JA, Leng S, Wang J, Fletcher JG, McCollough CH. Virtual monochromatic imaging in dualsource dual-energy CT: Radiation dose and image quality. Med Phys 2011;38:6371-9.

22. Doerner J, Hauger M, Hickethier T, Byrtus J, Wybranski C, Große Hokamp N, Maintz D, Haneder S. Image quality evaluation of dual-layer spectral detector CT of the chest and comparison with conventional CT imaging. Eur J Radiol 2017;93:52-8.

23. Meyer M, Haubenreisser H, Schoepf UJ, Vliegenthart R, Leidecker C, Allmendinger T, Lehmann R, Sudarski S, Borggrefe M, Schoenberg SO, Henzler T. Closing in on the K edge: coronary CT angiography at 100, 80 , and $70 \mathrm{kV}$-initial comparison of a second- versus a third-generation dual-source CT system. Radiology 2014;273:373-82.

24. Eckert J, Schmidt M, Magedanz A, Voigtländer T, Schmermund A. Coronary CT Angiography in Managing Atherosclerosis. Int J Mol Sci 2015;16:3740-56.

25. Hickethier T, Bar-Ness D, Bunck A, Maintz D, Pahn G, Coulon P, Si-Mohamed S, Douek P, Sigovan M. Noninvasive Evaluation of Soft-Plaque-Restenosis in Coronary Artery Stents: Initial In-vitro Comparison of a Spectral Photon Counting CT and a Spectral Dual-Layer CT System. Radiological Society of North America 2017 Scientific Assembly and Annual Meeting. Chicago, IL; 2017.

26. Hickethier T, Wenning J, Doerner J, Maintz D, Michels G, Bunck AC. Fourth update on CT angiography of coronary stents: in vitro evaluation of 24 novel stent types. Acta Radiol 2018;59:1060-5.

Cite this article as: Hickethier T, Wenning J, Bratke G, Maintz D, Michels G, Bunck AC. Evaluation of soft-plaque stenoses in coronary artery stents using conventional and monoenergetic images: first in-vitro experience and comparison of two different dual-energy techniques. Quant Imaging Med Surg 2020;10(3):612-623. doi: 10.21037/qims.2020.02.11 

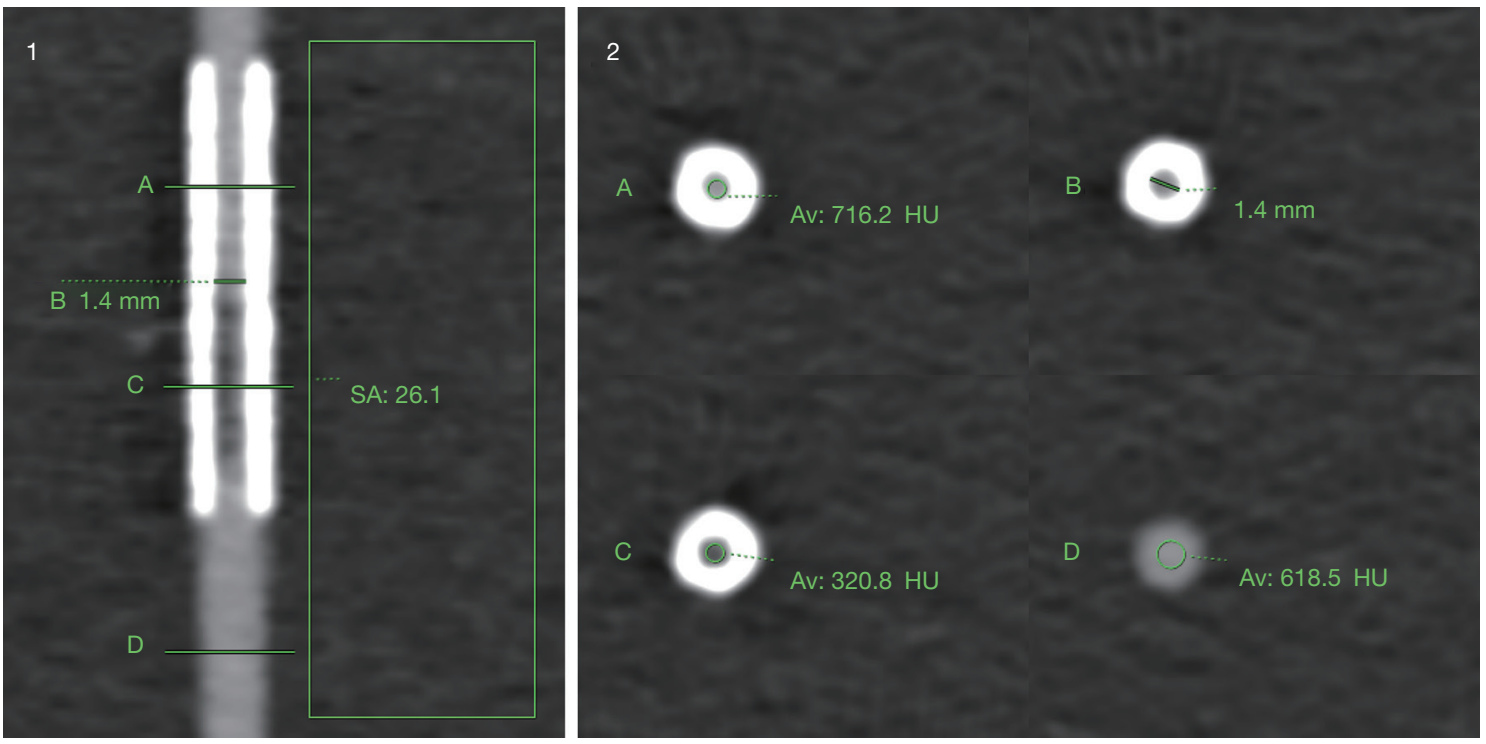

Figure S1 Example of quantitative measurements demonstrated on a $60 \mathrm{keV}$ DLCT monoenergetic reconstruction of a PlCr stent (“Omega”). 1 (left): longitudinal reformation with 4 measurement planes: A, in-stent attenuation; B, visual lumen diameter (measured in longitudinal and axial orientation); C, stenosis density; D, luminal attenuation. (right): image noise in a standardized ROI adjacent to the stent; 2: corresponding axial measurements to the 4 planes (A,B,C,D) described above. ROI, region of interest; DLCT, dual-layer computed tomography.

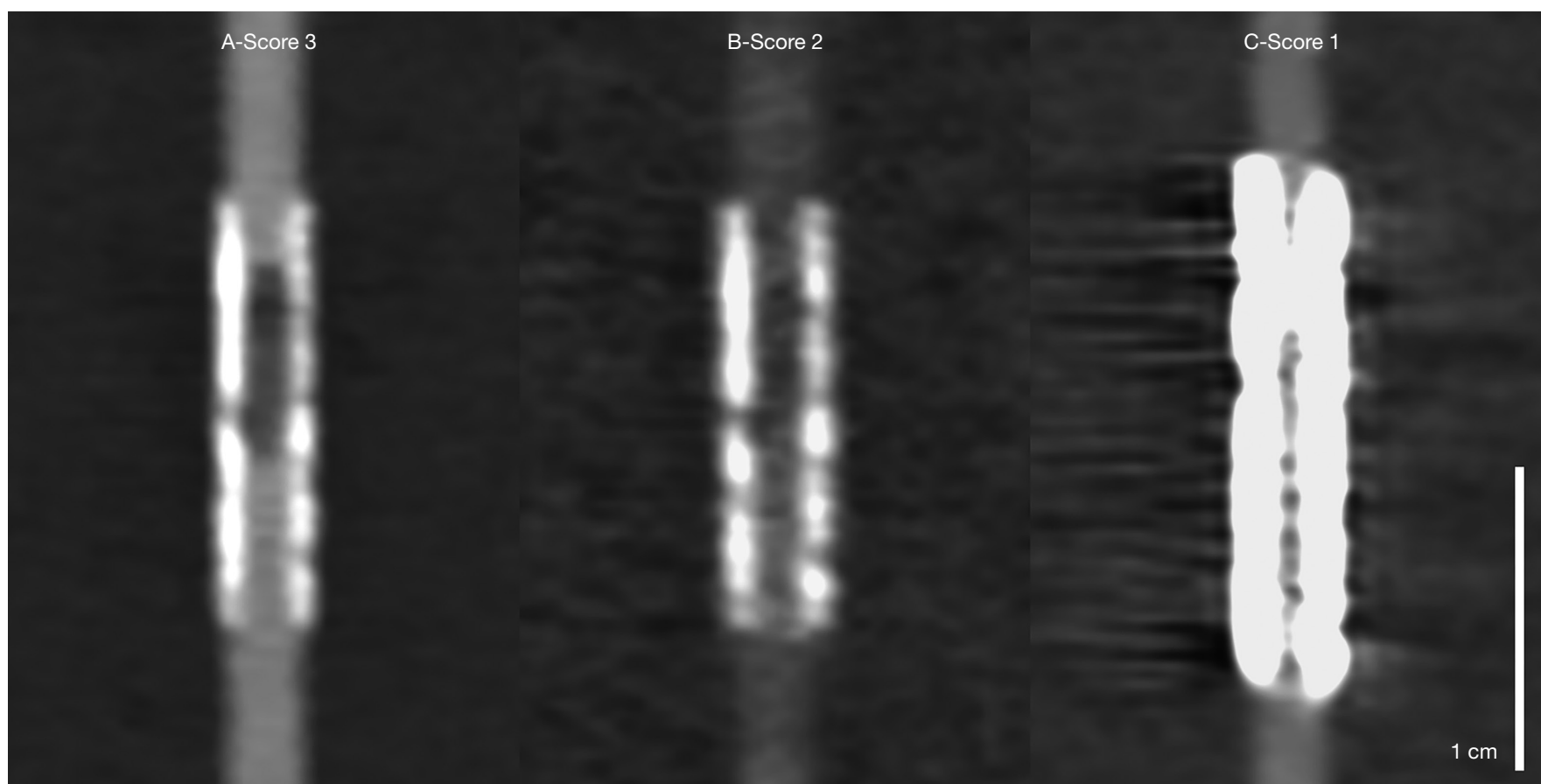

Figure S2 Example images of stents of the three different subjective scores given (score 4 and 5 were not given). (A) Score 3-stenosis and longitudinal extent clear, but residual lumen undistinguishable ("Pro Kinetic"; MonoE 40 keV, DLCT); (B) score 2-lumen appears stenosed with unclear longitudinal extent ("Pro Kinetic"; MonoE $100 \mathrm{keV}$, DLCT); (C) score 1—stent associated artifacts impede lumen assessment and stenosis not visible ("Tantal Coronary"; PolyE, DLCT). LCT, dual-layer computed tomography; PolyE, polyenergetic; MonoE, monoenergetic. 
Table S1 Results of density measurements inside the stent lumen and of the stenosis

\begin{tabular}{|c|c|c|c|c|}
\hline Reconstruction & \multicolumn{2}{|c|}{ Density inside stent lumen $(\mathrm{HU})$} & \multicolumn{2}{|c|}{ Density of stenosis (HU) } \\
\hline PolyE & $586.2 \pm 96.8$ & $531.5 \pm 168.9$ & $111.3 \pm 74.9$ & $220.4 \pm 150.6$ \\
\hline MonoE 40 & $1,677.2 \pm 305.4$ & $1,553.5 \pm 120.4$ & $430.2 \pm 120.5$ & $526.4 \pm 105.8$ \\
\hline MonoE 50 & $1,095.4 \pm 170.8$ & $1,069.8 \pm 184.9$ & $258.9 \pm 96.0$ & $383.0 \pm 140.6$ \\
\hline MonoE 70 & $566.6 \pm 84.7$ & $628.0 \pm 289.7$ & $129.0 \pm 82.6$ & $250.3 \pm 206.3$ \\
\hline MonoE 80 & $439.9 \pm 101.4$ & $498.3 \pm 236.3$ & $103.3 \pm 97.0$ & $229.7 \pm 229.9$ \\
\hline MonoE 90 & $365.0 \pm 132.6$ & $461.1 \pm 335.7$ & $85.0 \pm 102.5$ & $207.3 \pm 234.8$ \\
\hline MonoE 100 & $305.0 \pm 147.4$ & $410.7 \pm 345.2$ & $70.8 \pm 109.4$ & $184.9 \pm 243.1$ \\
\hline
\end{tabular}

DSCT, dual-source computed tomography; DLCT, dual-layer computed tomography; PolyE, polyenergetic; MonoE, monoenergetic.

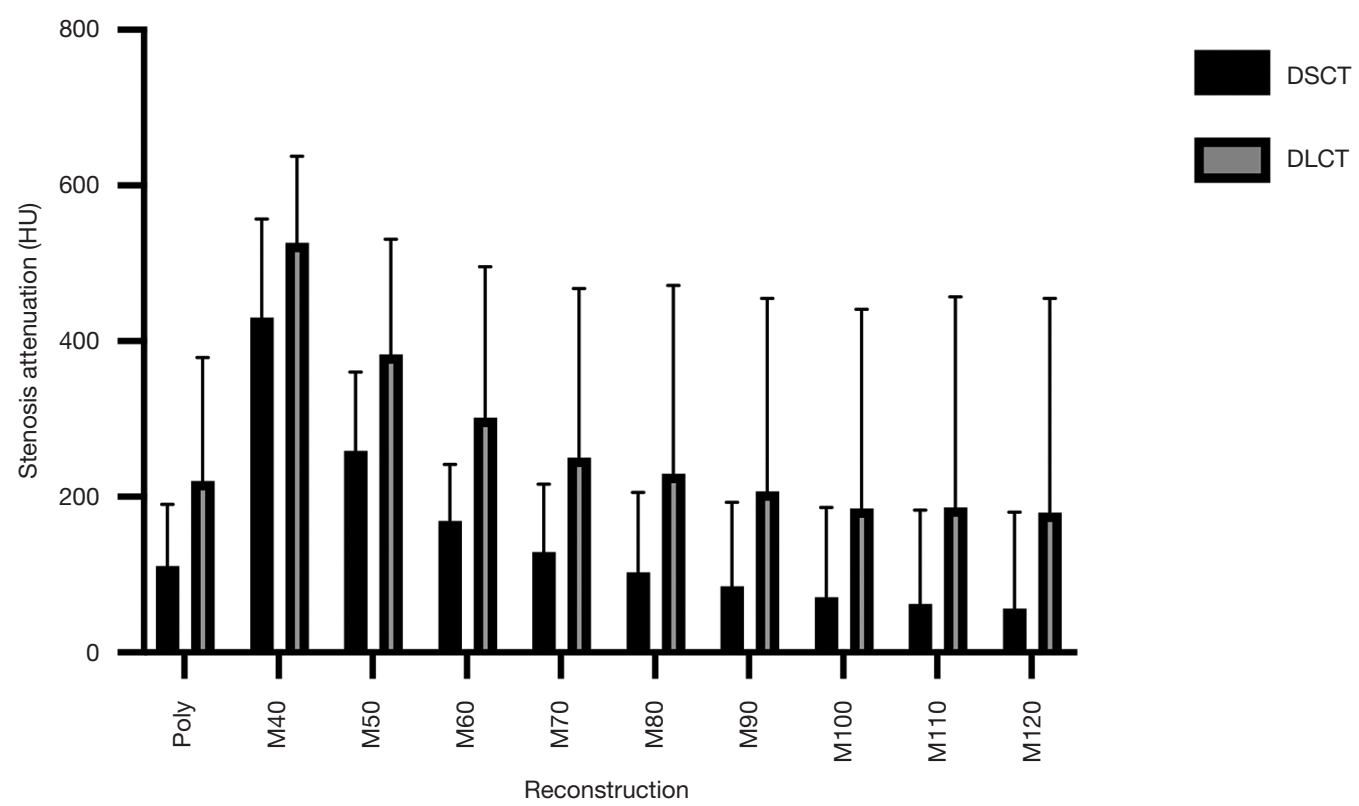

Figure S3 Graphical depiction of the density of the CT scanners used for the different reconstructions of the two different CT scanners. As described in the text, there is an increase in density in the low keV MonoE reconstructions compared to the PolyE and the high keV MonoE images. However, this was markedly less pronounced than the increase in density in the rest of the stent lumen. (see also Table S1). DSCT, dual-source computed tomography; DLCT, dual-layer computed tomography; PolyE, polyenergetic; MonoE, monoenergetic. 